\title{
“城市引擎” 规则建模在城市规划中的作用与特点
}

\author{
Functions and Characteristics of City Engine and Its Rule-based Modeling in Urban Planning
}

骆燕文 何江

Luo Yanwen, He Jiang

\begin{abstract}
摘要: “城市引擎”规则建模可以快速生成大量的模型, 在城市规划领 域有很大的应用潜力, 但目前国内外对其研究较少。本文从城市规划 角度出发, 在已有研究基础和实践案例上, 对规则建模的优势、模型 精细程度、建模步骤和如何辅助城市规划做了研究。在城市规划应用 方面提出规则建模主要有数据准备与处理, 城市建设要素类型分类, 规则编写与赋予, 模型生成和深化四个步骤。基于其建模精度, 适宜 应用的规划层次是总体规划、控制性详细规划。其在城市规划的辅助 作用主要体现在三维模型空间展现、数据输出和分析、辅助规划管理 和提供公共参与平台, 以及与其他软件结合辅助规划方案设计和管理。

Abstract: City Engine (CE) can efficiently generate 3D city models by rulebased modeling and has great potential in supporting urban planning and design. Nowadays there is still lack of research about City Engine in the urban planning field. From the viewpoint of urban planning, this study analyzes advantages of rule-based modeling, model accuracy and modeling steps based on previous studies and planning projects. From the above analysis, the present paper describes how to support urban planning and design. For the application of CE-based models to urban planning, the following four major steps are proposed in the paper: (1) data preparation and processing; (2) classification of urban construction elements; (3) generating and assigning rules; (4) model generation and modification. Under consideration of model accuracy, CE models are suitable for supporting the master planning and the regulatory detailed planning design. In urban planning, CE could play the following supporting roles: $3 \mathrm{D}$ visualization of urban space; data output for urban environment analysis, assessment and management; and providing a communication platform for public participation. It would also be combined with other application softwares for supporting urban planning and management.
\end{abstract}

关键词: 城市引擎; 快速三维建模; 城市规划; 辅助设计

Keywords: City Engine; Efficient Modeling; Urban Planning; Aided Design

国家自然科学基金项目名称：建筑一体化太阳能系统设计与分析技术 研究 (51368003)

作者: 骆燕文, 广西大学土木建筑工程学院, 博士研究生。 275654781@qq.com

何江 (通信作者), 博士后, 广西大学土木建筑工程学院, 教授。 kakohejiang@gxu.edu.cn

\section{引言}

中国自 20 世纪 80 年代末开始引人三维建模技术, 并已 经发展了快 30 年 ${ }^{[1]}$ 。城市规划界也相继提出了 “ “数字城市”、

“绿色城市”、“智慧城市”等与城市三维建模紧密相关的规 划理念。城市三维建模技术凭借自身的几何属性与相关信息 对有效控制城市资源、合理选择城市空间发展方向、正确进 行城市规划管理有很大辅助作用 ${ }^{[2,3]}$ 。随着可视化多维图像 的发展, 无论是设计人员、政府部门还是公众均对城市三维 模型的要求和需求越来越高 ${ }^{[1]}$ 。

在这样的背景下，欧美国家开始采用一种规则建模方法 实现快速批量建立城市模型。“规则”一词的原意是指规定 出来供大家共同遵守的制度或章程, 用于三维建模是指采 用一系列的计算机语言对模型进行描述, 并决定模型如何生 成 ${ }^{[4,5]}$ 。在建模过程中, 将规则赋予到城市基底上, 就可以 批量生成城市建设要素, 快速反映城市总体风貌。目前业界 采用规则建模的主流软件为 “城市引擎” (CE: City Engine)。 该软件采用的规则建模方法速度快, 可以输出简单定量数据, 在辅助城市规划设计和管理方面具有一定的潜力 ${ }^{[5,6]}$ 。国内 对 CE 规则建模的研究刚刚兴起, 已有研究多集中在介绍快 速建模特点和规则编写方面 ${ }^{[5,7,8}$, 对其建模优势、建模步骤、 模型精度描述以及怎样辅助城市规划还较少有文献涉及。本 文从城市规划角度出发, 对 CE 规则建模与传统建模方法做 了比较, 讨论其模型精细程度 ; 总结规则建模的主要步骤和 其在城市规划中的辅助作用。

\section{1 规则建模概况}

\section{1 技术背景}

采用规则进行建模的 CE 软件最初由瑞士苏黎世理工 学院于 2001 年开发, 在经历了多次升级和授权后, 目前是 
Esri 公司旗下的产品 ${ }^{[9,10]}$ 。Esri 公司主要产品为 ArcGIS（获 取、分析城市地理信息数据的主要软件), 有共同平台的优 势促使 CE 与 ArcGIS 可实现无缝连接。CE 建模原理是利用

“Computer Generated Architecture” (简称 CGA) 语言对建 模要素进行语句描述, 这一系列语言称之为 $\mathrm{CE}$ 的规则 ${ }^{[11]}$ 。 一个规则（也可称为规则文件）包括规则函数、属性、自定 义函数和注释。常用的函数包括拉伸、合并、上色、拆分; 属性是一组静态的全局变量, 每个属性被初始化为一个特定 的值; 自定义函数可以为带参化、标准化、随机化、条件化、 递归化的语法描述 ; 注释是为了提高规则的易读性。图 1 展 示了如何利用规则在一片空地上快速生成三维模型。这个案 例主要采用了基本函数、自定义函数对建筑物的高度、屋顶 形式、色彩、建筑密度以及绿地进行定义。将编辑好的规则 赋予到指定地块内, 点击模型生成命令, 则在地块内生成规 则描述的城市景观。与传统交互式建模最大的区别在于规则 决定当前建模对象的变化过程, 并把变化的结果赋予到一个 或多个对象 ${ }^{[12]}$, 实现批量快速的生成三维模型。CE 基于规 则的批量建模可以实现快速创建城市三维模型、输出模型数 据、网络共享模型等功能, 与传统建模相比有一定的优势, 是未来城市三维建模的一个趋势 ${ }^{[13,14]}$ 。

\subsection{CE 规则建模的优势}

现有的城市三维建模一般采取以下三种方法 : 一是基 于规划图纸等二维数据, 利用三维建模软件 (Sketch Up、 3DMAX 等) 进行交互式建模; 二是利用 GIS 的二维线划 数据及其相应的高度属性进行三维建模; 三是利用数字摄 影丈量技巧进行三维建模 ${ }^{[15]}$ 。目前直接利用第一种方式一 建模软件交互式精细建模最为普遍。传统精细三维建模方 式虽然展示效果好, 但其结果往往是静态、固化的模型, 仅能够用于立体视觉表达, 并不能满足属性查询、三维空 间分析等深层次的应用, 且很难重复利用 ${ }^{[5,16]}$; 同时建模的 时间和劳动成本高, 无法快速生成模型。对于城市建模而言, 采用 $C E$ 规则建模与已有的建模方式相比, 具有以下四点 优势。

（1）建模速度快。规则建模初期需要更多的时间来编写
规则, 但一旦完成该工作, 就可以在具有共性的建设对象上 批量创造模型。城市的规模越大, 规律性越高, 越能体现规 则建模速度快的优点。传统建模方式普遍耗时耗力, 成本与 城市规模成指数正比关系。

(2) 可较为轻松地反复修改和细化模型。规则编辑后保 存, 可以在已有基础上对定义语句反复优化设计, 达到较易 修改模型或创造更多细节的目的 ${ }^{[17,18]}$ 。已有建模方式修改和 细化模型花费时间与新建模时间持平甚至更高, 特别是对于 整个城市的大规模模型修改, 往往需要耗费很大的精力才能 达到预期效果。

(3) 可输出简单的模型数据。通过 CGA 语言描述输出 对象, 在 CE 自带报表栏可输出想要的模型数据, 例如树木 面积、数量、建筑高度等。城市规划若能在三维空间实现城 市信息半定量化, 对公众而言则提高了规划的理解和公信力。 传统建模方法还很难实现城市建设数据输出功能。

(4) 可移植共享。编写规则的经验可以继承, 同时编写 的规则文件也可共享。规则文件可以积累为规则库, 起初使 用和编写得越多, 积累的规则文件越多。对有共性的对象可 以直接从规则库找到相应规则进行应用, 对有差异性对象可 以对已有规则进行语句修改。由于每个城市风貌不同, 而传 统建模方式几乎是纯手动交互式建模, 故新方案均是重头开 始, 极少能在已有旧模型上重复利用。

除了以上四点基于规则编写而具有的优势, 相比较传统 建模方式, 模型可在公共网络进行联动共享查看。

\section{3 精度描述与在城市规划中的适宜应用范围}

$\mathrm{CE}$ 规则建模能快速地反映城市风貌, 但三维模型不如 传统手动交互式建模精细 ${ }^{[19,20]}$ 。其使用优势范围如图 2 所示, 建模价值与城市规模成正比 : 城市规模越大, 对细节要求越 低, 规则可重复利用率越高, 价值越高; 城市规模越小, 对 细节的要求越高, 规则可重复利用率越低, 价值降低。当展 示 1:10 000 1:1 000 的城市风貌时, CE 规则建模可以将城市 总体肌理、路网结构、不同性质用地的特色、城市天际线、 绿地系统等大面积建设要素完整的反映出来 (图 3)。当展 示 1:100 1:1 000 的城市风貌时, 建筑细部、植物搭配层次,

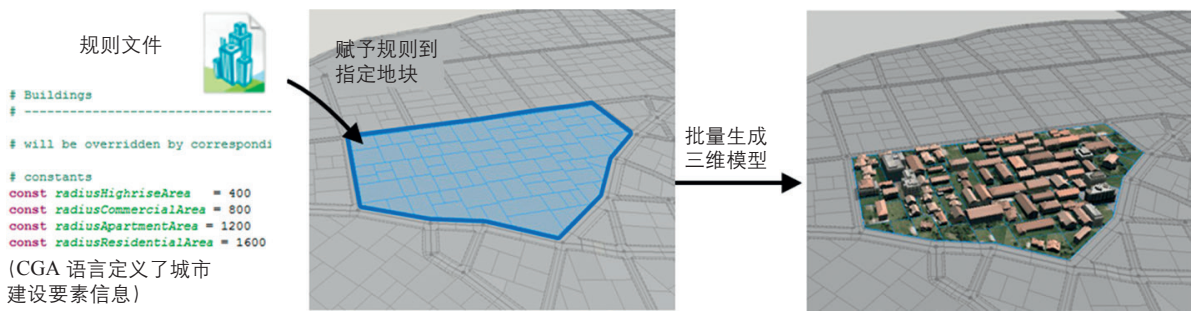

图 1 对城市建筑物等的 CE 规则建模示意图 资料来源 : 作者绘制

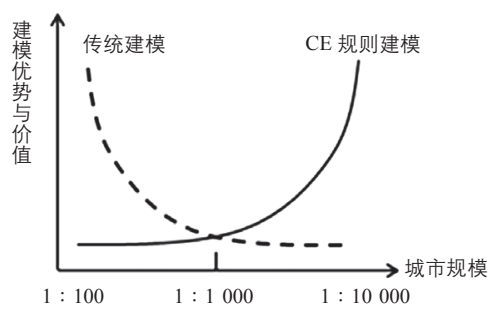

图 2 CE 规则建模优势与传统建模相比 资料来源：作者绘制 
甚至是生活场景等细节需要更多更复杂的规则以及贴图纹理 数据才能实现（图 4)。因此对于城市标志性建筑物或场景, 建议在传统软件完成精细建模后再导人 CE 建立的城市场景, 方可较完整地实现城市三维模型。

基于 $\mathrm{CE}$ 规则建模的精细程度, 本文认为该建模方法更 加适用于城市规划中的总体规划和控规性详细规划（简称控 规)。对于小范围的城市建模, 如修建性详细规划以及建筑 设计层次的建模, $\mathrm{CE}$ 规则建模花费的时间和劳力成本与传 统手工建模相比优势并不特别明显。因此对于小而精的建模 项目, 建议在 $\mathrm{CE}$ 中完成基础体块拉升后导人传统建模软件 中进行精细加工。

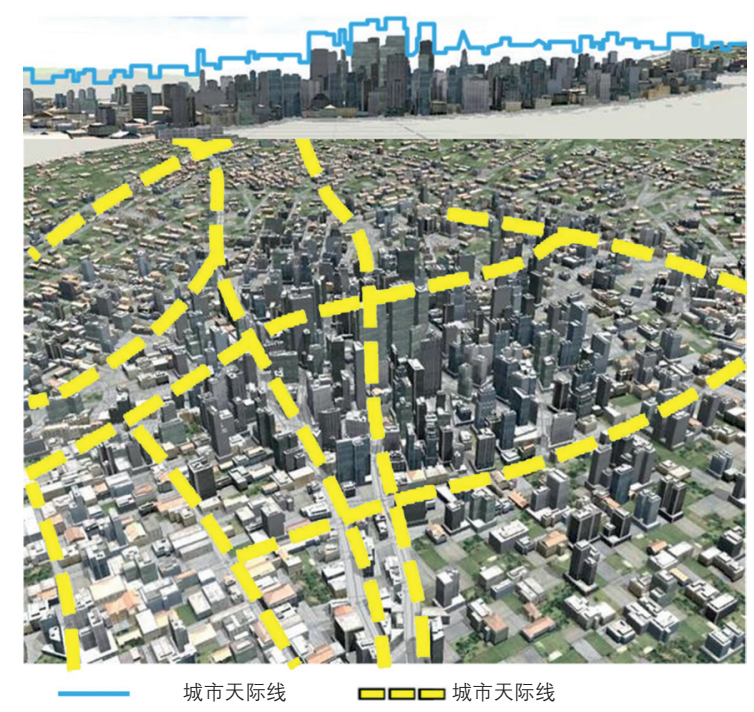

图 $3 C E$ 规则建模展示 1:5000 城市风貌 资料来源 : 作者绘制

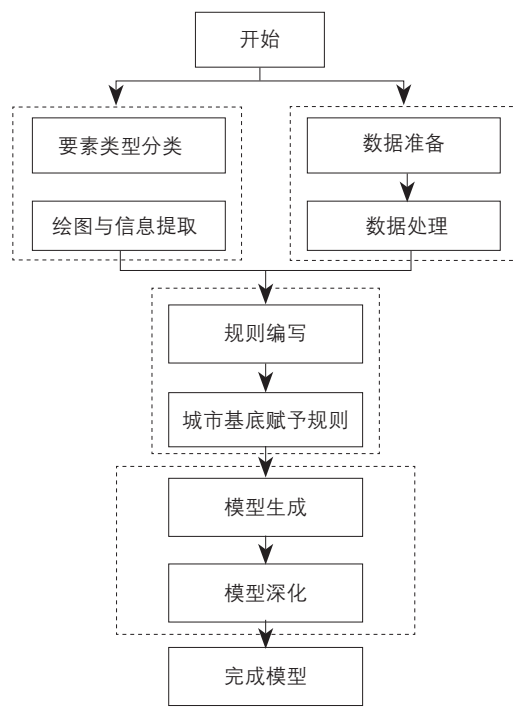

图 6 CE 规则建模主要步骤 资料来源 : 作者绘制

\section{2 城市规划中的规则建模}

\section{1 技术思路}

通过对国外已有实践案例的研究, 如法国马赛、北京 奥运村（图 5) 等, 同时结合城市模型特点, 本文归纳基于 $\mathrm{CE}$ 的规则建模有以下四个关键步骤: 数据准备与处理、城 市建设要素类型分类、规则编写与赋予、模型生成和深化 (图 6)。这四个步骤从城市角度出发, 对前期建模基础一 城市基底、中期最大化利用规则优势和编写规则, 以及后期 生成和模型深化做了解释, 是 $\mathrm{CE}$ 实现三维城市模型的主要 步骤。

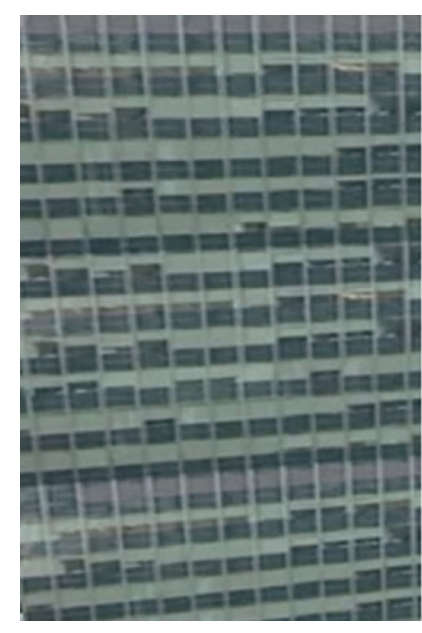

$1: 5000$ 贴图形式建筑立面精度低，规则简单 图 4 CE 规则建模在不同尺度城市模型中的建筑细部效果 资料来源 : 作者绘制

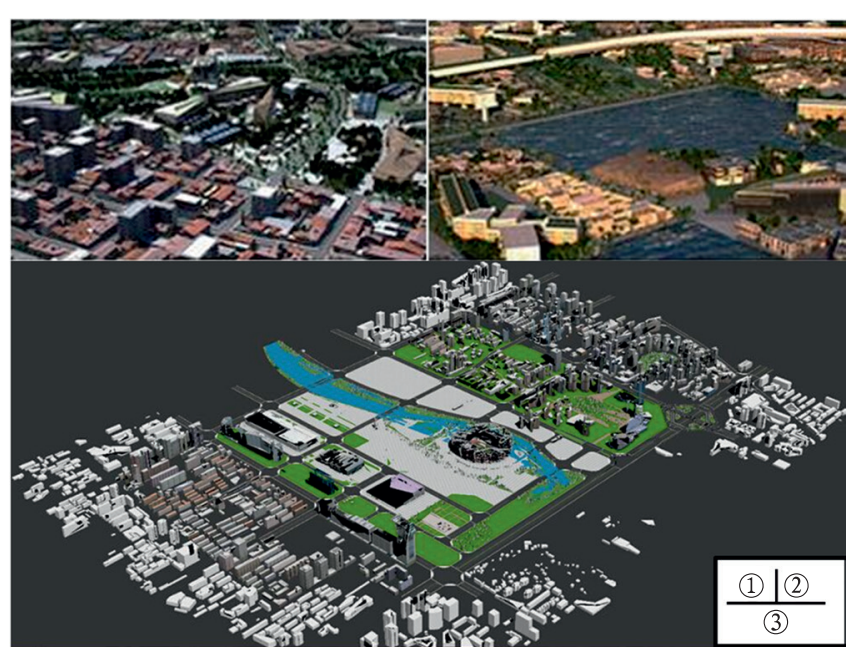

注: (1)和(2)马赛, (3)北京奥运村

图 5 CE 规则建模建立的城市三维模型

资料来源 : http://blog.3snews.net/space.php?uid=53325\&do=blog\&id=62597 


\subsection{CE 规则建模步骤}

(1) 数据准备与处理

构建城市模型需要准备的基础数据非常多, 包括地形 数据、建筑物基地投影数据、交通道路设计、构建物几何 数据、贴图纹理数据等。其中地形数据、交通道路数据和 建筑物基地投影数据基本能完整的反映城市基底情况, 是 实现三维场景的基础。这三种数据一般为 $\mathrm{dxf}$ 格式的数据, 需要通过 ArcGIS 进行点线面转换后保存为 Shapefile 或 File Geodatabase 格式 ${ }^{[21,22]}$ 。由于 CE 中只能设置投影坐标系, 因 此还需要对建模的数据进行投影转换。构建物几何数据是依 据城市实际风貌、城市设计、规划控制要求等, 对空间位置、 形态、高度等做数据收集。贴图纹理数据是以二维图片形式 进行收集, 获取方式有实地拍照、航空摄影、数字全景摄影 等。几何数据和贴图数据越详细丰富、越接近真实城市风貌, 后期三维模型则越逼真鲜活。

(2) 城市建设要素类型分类

该步骤是本文基于城市形态设计准则思想提出 : 对城市 中心到边界进行立面和平面设计，划分若干类城市风貌。再 提取城市风貌的基本类型, 为规则编写提供依据（图 7)。具 体做法是依据卫星影像地图和实地调研, 结合设计方案, 将 各个不同区域的街道、建筑、绿化等进行形态特征分类，并 在交界区进行形态过渡设计 ${ }^{[23,24]}$ 。将分好类的城市风貌的建 设要素信息进行提取，再进行绘图（包括立面、平面）并标 注基本的要素尺寸。这个工作将城市风貌进行归类，为规则 编写提供依据, 实现一个规则可以重复应用于风貌相同的多 个地块的目的。

（3）规则编写与赋予

依据城市建设要素类型分类, 采用 CGA 语言对对象进 行描述和规则编写。利用若干个基本规则表述城市建设基本 类型, 再依据实际情况做语句修改变化细节, 则可一定程度
快速反映城市总体面貌。该方法将规则批量建模特点最大化 利用, 大大减少了城市建模的时间和劳力成本。当为城市总 体规划建模时, 初略的规则描述即可反映城市总体风貌; 当 为城市详细规划建模时, 需依据建筑几何数据、道路设计等 要求对规则进行详细描述。具体的语言编写本文不作详细介 绍, 可参考文献 $[4,5,18,25,26]$ 。

（4）模型生成和深化

将处理过的地形、交通道路、建筑物基底投影等数据导 人 $\mathrm{CE}$ 软件, 生成城市地面肌理。将编辑好的若干基本规则 分别赋予不同的城市基底中, 生成初步城市三维模型。对初 步模型进行贴图和纹理处理后就基本能反映城市风貌。再通 过手动交互式模型调整和检视窗口模型调整操作的配合, 可 对模型的高度、色彩、形式进行细微改动, 体现城市的多元 化, 实现鲜活的城市模型。

\section{CE 规则建模在城市规划中的辅助作用}

$C E$ 规则建模一定程度上可以辅助城市规划设计, 主要

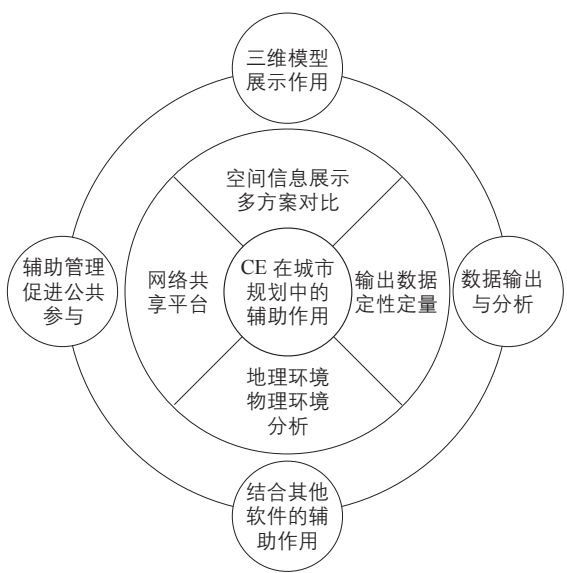

图 $8 \mathrm{CE}$ 规则建模在城市规划中的主要辅助作用 资料来源：作者绘制

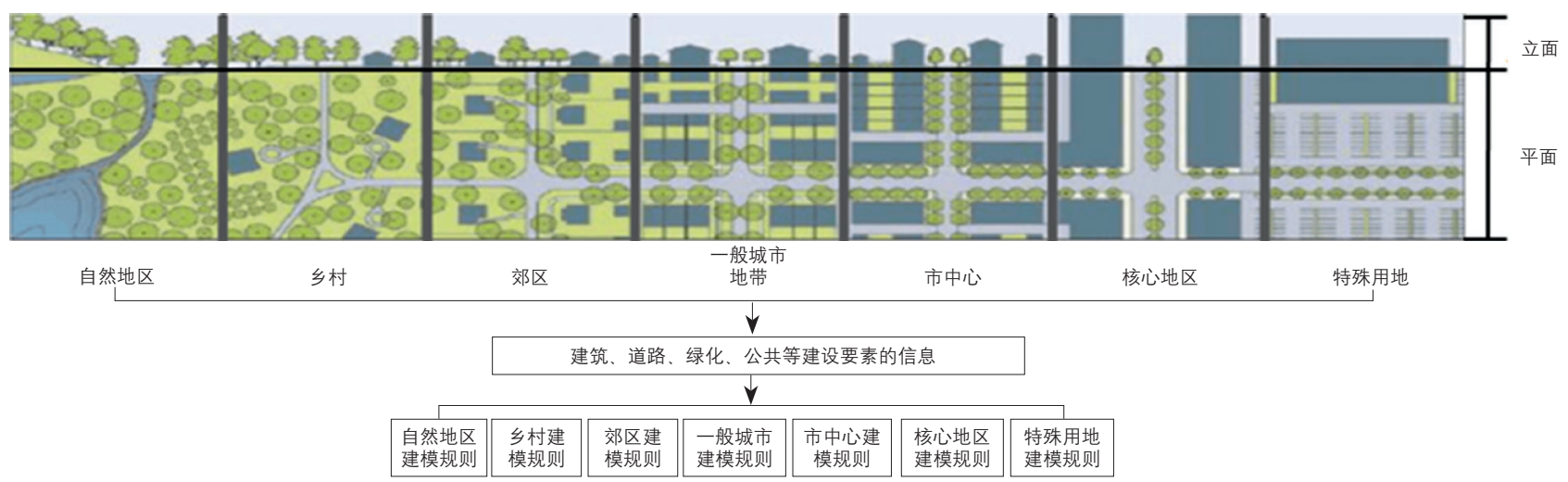

图 7 利用城市形态设计准则思想的城市建设要素分类 资料来源 : 作者在形态准则控制框架图的基础上绘制 
包括: 三维模型自身展示功能辅助方案设计; 输出简单的数 据辅助分析生态建设平衡和利益权衡; 三维模型的公共网络 展示平台辅助规划管理和促进公众参与 ; 与其他软件的结合 能拓展对城市模型的分析, 增加辅助功能 (图 8)。

\section{1 三维模型展示作用}

(1) 城市总体

完整的城市三维模型可以多视角分析城市的空间和结构 特征, 还可以为下一层级的规划作建设指导, 在总体规划编 制中具有一定的意义 ${ }^{[27]}$ 。由于成本大, 且不是法定内容, 所 以目前我国在城市总体规划编制过程中一般不会做城市建模 工作。只有个别城市开始尝试总体规划实现三维模型, 如武 汉市正在推进的智慧城市总体规划要求进行三维城市模型报 建和展示 ${ }^{[28]}$ 。总规阶段的三维模型需要反映城市总体风貌和 结构空间关系, 对城市建设要素细节不作特别要求, 故 CE 能快速实现城市大致风貌的建模特点非常适合城市总体规划 大尺度建模的需求。

通过规则编写可以批量拉伸建筑体块和赋予纹理材质, 快速建立不同特色的城市片区、整体控制城市天际线、划分 绿地系统的三维模型。这样建立的模型足够展示城市总体风 貌, 同时辅助分析和协调空间结构、城市功能和城市形态三 者之间的关系。例如新建城市巴西利亚城市案例, 建成后获 得襄扬的同时也遭到了很多批评。一方面, 强调纪念性和形 式性意味着让人远离而不是让人亲近; 另一方面, 功能主义 认为建筑满足通风、日照、卫生等技术要求即可, 因而追求 建筑低密度、高层数, 丧失了尺度感 ${ }^{[29,30]}$ 。若在总体阶段实 现可灵活操作的城市三维模型, 就可多视角多方位地感受按 照飞机形态进行功能划分的城市各片区建设在色彩、尺度、 风格方面是否协调; 感受公共服务设施、自然生态环境与各 功能片区之间是否能频繁互动; 还能从行人角度体验城市地
面空旷、街道宽阔、建筑高䇯的空间尺度。

城市三维模型可以更细致和鲜活地展示总体规划二维平 面的设计内容, 反馈总体规划的预期规划效果, 为总体规划 提供一个更感性和人性的思考方式。

\section{(2) 控规性详细规划}

控规处于总体规划和修建性详细规划之间, 它填补了形 态示意的缺陷, 最大限度实现规划的可操作性, 因此三维模 型在控制性详细阶段是非常重要的 ${ }^{[31]}$ 。控规中三维模型的主 要任务是：体现定量的建设控制性内容：为共性控制提供标 准建设模型; 还要体现定性的建设引导性内容 : 为个性设计 提供多种可能。传统建模方式固态展示城市环境, 只能大致 反映控规指导下的城市环境。

CE 规则建模可以通过将强制性和引导性控制指标编人 规则中真实反映控制下的城市建设环境。例如对定量的强 制性控制指标规则编写: 控制某地块容积率为 2.5 , 将该指 标进行规制编写描述该地区容积率为 2.5 , 则在三维模型中 无论作怎样的调整, 该地块的容积率均为 2.5 。对引导性控 制指标的规制编写：引导某片区建筑屋顶为坡屋顶或平屋 顶，对该指标进行规制编写时描述该片区屋顶为坡屋顶和 平屋顶两种形式, 但可随机选取。则该片区建筑屋顶统一 为平屋顶或坡屋顶, 但建筑可以随机选取两种屋顶样式的 任意一种。通过在视检窗口进行调整操作, 还可以多次随 机调整屋顶形式。同样道理, 可以将土地使用控制、建筑 高度和色彩控制、城市设计引导的相关指标等通过规则编 写反映到三维模型中, 最终将控规的内容用三维模型真实 地展现出来。因此 CE 规则建模在控规阶段具有很大的辅 助潜力。但目前用规则完整地将一个控规方案的指标表述 出来还比较困难, 主要是指标太多, 规则编写的工作量会 非常庞大, 加上很多指标之间存在联系, 规则的编写就容 易出错。

\section{表 1 CE 报表输出数据与其辅助作用}

\begin{tabular}{|c|c|c|c|}
\hline 类型 & 输出数据 & 辅助分析 & 作用 \\
\hline \multirow[t]{4}{*}{ 绿化 } & 植物总数量 & 生态效益、绿化遮阳 & \multirow{4}{*}{$\begin{array}{l}\text { 权衡生态环境、城市建设与市民 } \\
\text { 利益三者的关系 }\end{array}$} \\
\hline & 不同植物种类数量 & 景观搭配、绿化遮阳 & \\
\hline & 绿地地块数量 & 景观廊道、栖息地串联 & \\
\hline & 绿化面积 & 生态效应、市民福利 & \\
\hline \multirow[t]{3}{*}{ 建筑 } & 色彩种类 & 色彩协调 & \multirow[t]{3}{*}{ 控制城市风貌、历史街区保护 } \\
\hline & 建筑高度 & 城市天际线 & \\
\hline & 屋顶、立面等样式数量 & 城市特色 & \\
\hline \multirow[t]{3}{*}{ 公共设施 } & 路灯数量 & 道路夜间照明 & \multirow[t]{3}{*}{ 合理分配和布置公共设施 } \\
\hline & 垃圾桶数量 & 城市环境卫生 & \\
\hline & 休息板発数量 & 休憩设施 & \\
\hline
\end{tabular}

参考文献 : 作者依据参考文献 $[12,19,32]$ 整理 
$\mathrm{CE}$ 规则建立的三维模型, 可以一定程度上展示控规的刚 性要求和弹性要求, 为控规提供了一个直观真实的表述方式。

\section{2 数据输出与分析}

$\mathrm{CE}$ 可实现采集三维模型空间数据、提取建设对象信息, 并能输出简单数据, 为城市规划定量研究提供一些支持。现 有的城市规划虽然会有一些数据指标, 但无法衡量规划效果。 例如城市绿地率指标只对二维平面上的用地面积作规定, 但 在实际绿化建设中怎样种植植物, 种植什么植物等问题都直 接影响城市的生态和环境效果。

CE 数据输出也是通过规则编写实现：通过 CGA 语言定 义需要输出的数据, 就可以在报表工具栏输出相应的数据。 以绿化、建筑、公共设施四类建设信息为例, 表 1 展示了 CE 报表输出这三种类型的部分数据和可能起到的辅助作用。 通过这些数据信息, 可以具体地分析规划效果。如国外有学 者将生态效益与 CE 建立模型进行结合, 输出简单数据让决 策者和公众可以通过可视化的途径平衡生态建设与城市规划 之间的关系 ${ }^{[19,32]}$; 还有学者通过 CE 输出的建筑高度、色彩 种类和建筑样式的数据, 对城市风貌进行控制分析 ${ }^{[12]}$; 而对 公共设施的数据输出, 可以更合理地分配和布置公共设施。

简单的城市建设数据, 如数量、面积、百分比等, 可以 在初步设计中辅助判断城市建设效益和权衡各种利益, 对城 市规划设计和管理有一定的辅助作用。

\section{3 辅助规划管理和促进公共参与}

通过 CE 规则建模完成的三维模型可以在网络实现联动 编辑、方案比较、共享模型等功能, 为决策者和公众提供一 个易于管理和参与的平台。

该软件具备将模型发布到 Web 场景的功能, 可以通过 本地的 Web Scene Viewer 打开, 也可以发布到 ArcGIS Online 云端进行共享。打开火狐或谷歌汶览器, 输人调用链接地 址, 就可以在网络上看到规划后的三维模型。在 Web 场景里, 可以查询特定的建筑物或房间, 系统将进行自动定位; 还提 供日照分析功能, 可以计算某个时间的日照情况或模拟一天 的日照变化; 同时提供卷帘功能, 拉动卷帘可以关闭或显示 某个图层的信息, 实现对比的效果。

这些功能可以让管理者和公众简洁直观地了解规划方 案, 从多个层面和角度了解设计意图, 提高管理效率和公众 参与的范围和深度。

\subsection{CE 结合其他软件的辅助作用}

CE 建好的模型可以导人其他软件进行二次分析，拓展 更多功能辅助城市规划。例如与 ArcGIS 结合实现地理环境
分析; 与数值分析模拟软件结合实现城市物理环境分析 ; 与 虚拟现实系统结合模拟真实规划环境。

CE 与 ArcGIS 软件结合可以对城市进行地理环境分析 : 把模型导人 GIS 数据库中, 使用由 ArcGIS 的 ArcGIS 3D 分 析扩展模块提供的地理处理能力进行深人分析, 可以实现地 形数据的存储、查询、统计、分析、模拟、决策和预测等功 能 ${ }^{[32]}$ 。CE 完成的三维模型还具备导人其他软件进行物理环 境分析的潜力。例如可以导人数值模拟软件进行日照、风环 境等物理环境模拟。目前作者正在尝试将模型导人 Ecotect 和 CFD 等软件进行风环境、热环境等模拟, 有望实现更精 确的城市物理环境模拟。此外, 城市三维模型与虚拟现实系 统的结合也可让公众真实地体验城市环境。将城市模型导人 虚拟现实系统, 利用虚拟现实系统平台可以实现视觉、听觉、 触觉等感官的模拟, 让参与者如同身临其境一般及时、没有 限制地观察三维空间内的事物。

城市规划人员通常希望在一个三维模型中实现多种用 途, 而 CE 建模可以满足这个要求, 是当下众多建模软件中 实现一模多用的优选软件。

\section{4 结论}

综上所述, 由于 CE 规则建模可编写、修改和反复利用, 相比传统三维建模软件更适宜城市规模。规则建模能快速展 现城市总体风貌, 适宜在城市总体规划、控规中应用。其城 市建模步骤主要有四个, 分别为数据准备与处理、城市建设 要素类型分类、规则编写与赋予、模型生成与深化。其中基 于形态准则的城市建设要素分类使得若干个基本规则就可以 反映城市基本风貌, 充分发挥了规则建模快速建模的优势。 $C E$ 规则建模在城市规划中有一定的辅助作用, 主要为三维 模型提供空间信息，更好地展示总体规划、控规规划效果以 及实现多方案对比。可输出简单数据, 为城市规划定量研究 提供支撑。通过网络实现联动编辑、方案比较、共享模型等 功能, 为决策者和公众提供一个易于管理和参与的平台。与 其他软件结合可实现地理环境和物理环境分析。但目前 $\mathrm{CE}$ 的规则建模还存在一定的缺点 : 一是 CGA 语言编写并不是 非常容易掌握, 需要专业学习; 其次, CE 对于城市规划的 辅助作用初见锋芒, 但若想应用自如地辅助城市规划还需要 对规则进行更加简化和直观的设计, 方便规划设计人员进行 操作; 最后, 规则数据库是用得越多积累越多, 但目前我国 对 CE 的应用还处于起始阶段, 所以规则库的积累还不够。 随着 CE 在城市规划中的发展, 其在城市规划中的应用将逐 渐发挥其最大特点, 实现快速展示规划效果, 并在一定程度 上辅助规划设计。UP 


\section{参考文献}

[1] 刘玉洁. 城市三维模型及其在城市规划中的应用 [D]. 天津师范大学, 2014.

[2] 杨剑, 张云青, 潘伯鸣. 面向城市规划的三维建模技术探讨 [J]. 现代测 绘, 2003(1): 122-131.

[3] Steino Nicolai, Yildirim, Miray Ba, Ozkar Mine. Parametric Design Strategies for Collaborative and Participatory Urban Design [C]. 31st International Conference on Education and Research in Computer Aided Architectural Design in Europe, 2013. 1: 195-203.

[4] 张晖, 刘超, 李妍, 等. 基于 CityEngine 的建筑物三维建模技术研究 [J]. 测绘通报, 2014 (11) : 108-112.

[5] KyoHyouk Kim, John P Wilson. Planning and Visualising 3D Routes for Indoor and Outdoor Spaces Using CityEngine[J]. Journal of Spatial Science, 2014, 60(1): 179-193. 谢年, 向显, 徐艇伟. 基于规则的快速三维建模技 术研究 [J]. 城市勘测, 2013, (4): 5-8.

[6] 谢年, 向显, 徐艇伟. 基于规则的快速三维建模技术研究 [J]. 城市勘测, 2013, (4): 5-8.

[7] 朱以洲. 基于 CityEngine 的城市快速三维建模技术研究 [J]. 现代测绘, 2014, 42: 43-44.

[8] 花利忠, 王赵兵, 邹丽妹, 等. 基于 CityEngine 与 ArcGIS Flex API 的 校园 WebGIS 系统—以厦门理工学院为例 [J]. 厦门理工学院学报, 2013(4): 57-61.

[9] 梁世文, 尹志永, 李雪飞. CityEngine 在三维城市建设中的应用 [J]. 科 技传播, 2014 (05): 168-170.

[10] 周玲. 基于参数化技术的数字城市三维建模方法 [D]. 浙江大学, 2013.

[11] 施天越. 城市引擎软件在城市设计应用中的初步探讨 [J]. 建筑与文化, 2013(7): 98-99.

[12] Barroso S, Besuievsky G, Patow G. Visual Copy \& Paste for Procedurally Modeled Buildings by Ruleset Rewriting[J]. Computers \& Graphics, 2013, 37: 238-246.

[13] CityEngine 为 GIS 应用开启一种新的方法 [EB/OL]. (2011-11-09) [2015-12-05]. http://www.esrichina.com.cn/2011/1109/1548.html.

[14] Watson Benjamin, Mueller Pascal, Wonka Peter. Procedural Urban Modeling in Practice[J]. IEEE Computer Graphics and Applications, 2008, 28(3): 18-26.

[15] 官云兰, 周世健, 张明. 基于 ERDAS IMAGINE 的城市三维景观构建 [J]. 江西科学, 2007(4): 454-457.

[16] 李鸿祥. 三维城市规划辅助决策支持系统的设计与实现 [D]. 厦门大学, 2013.

[17] Zhao X, Coors V. Combining System Dynamics Model, GIS and 3D Visualization in Sustainability Assessment of Urban Residential Development[J]. Building and Environment, 2012, 47: 272-287.

[18] 吕永来, 李晓莉. 基于 CityEngine 平台的高速铁路建模方法的研究与 实现 [J]. 测绘, 2013, 36(1): 19-22.

[19] Neuenschwander N, Wissen U H, Grêt-Regamey A. Integrating an Urban Green Space Typology into Procedural 3D Visualization for Collaborative Planning[J]. Computers, Environment and Urban Systems, 2014, 48: 99-100.

[20] Mourao Moura, Ana Clara. Geodesign in Parametric Modeling of Urban Landscape[J]. Cartography and Geographic Information Science, 2015, 60(1): 179-193.

[21] 王俊伟, 简季, 周云帆. 数字校园应用中 CityEngine 快速参数化三维建 模研究 [J]. 测绘, 2014(4): 161-164.

[22] Dahal K R, Chow T E. A GIS Toolset for Automated Partitioning of Urban Lands[J]. Environmental Modelling \& Software, 2014, 55: 222-234.

[23] 王晓川. 精明准则一一美国新都市主义下城市形态设计准则模式解析 [J]. 国际城市规划，2013(6): 82-88.

[24] 高晖. 美国城市形态设计准则的启示及应用 [J]. 规划师, 2014(S5): 210-214.

[25] 江涛. 基于 City Engine 的虚拟校园研究与实现 [J]. 西安文理学院学报 (自然科学版), 2014(4): 81-74.

[26] 朱安峰, 王海鹰, 高金顶. 基于 CityEngine 的三维数字校园系统 [J]. 计 算机系统应用，2015(2): 112-115.
[27] 牛强, 宋小冬, 周婕. 基于地理信息建模的规划设计方法探索—— 以 城市总体规划设计为例 [J]. 城市规划学刊, 2013(1): 90-96.

[28] 翁志勇, 江城, 白应兵. 智慧武汉建设规划及武汉智城的实践 [J]. 智能 建筑, 2013(6): 59-66.

[29] 谭源. “乌托邦” 的终结: 从昌迪加尔及巴西利亚规划谈起 [J]. 南方建 筑, 1999(4): 83-84.

[30] 李玉峰. 新遗产城市 [D]. 中央美术学院, 2010.

[31] 储征伟, 陈昕. 三维城市模型 (3DCM) 在控制性详细规划编制中的应 用研究 [J]. 城市勘测, 2008(1): 21-23.

[32] Grêt-Regamey A, Celio E, Klein T M, et al. U W. Understanding Ecosystem Services Trade-offs with Interactive Procedural Modeling for Sustainable Urban Planning[J]. Landscape and Urban Planning, 2013, 109: 107-116.

(本文编辑：许玫) 